\title{
DETERMINAÇÃO DE REGIÕES HOMÓLOGAS PARA REGISTRO DE UMA SÉRIE MULTITEMPORAL DE IMAGENS DE SATÉLITE USANDO ALGORITMOS GENÉTICOS
}

\author{
Establishing of homologous regions for the registration of a multitemporal series of \\ satellite images by using genetic algorithm \\ MARA RUBIA SILVA \\ JORGE ANTONIO SILVA CENTENO \\ HIDEO ARAKI \\ Universidade Federal do Paraná \\ Setor Ciências da Terra, Departamento de Geomática \\ Curso de Pós-Graduação em Ciências Geodésicas, Curitiba -PR \\ \{mara_silva, centeno, haraki\}@ufpr.br
}

\begin{abstract}
RESUMO
O presente trabalho faz parte do programa GEOSAFRAS, estimativa e safras no Brasil, coordenado pela Companhia Nacional de Abastecimento e do Programa das Nações Unidas para o Desenvolvimento. Uma aplicação muito comum no gerenciamento de safras é o monitoramento de mudanças nos campos de cultivo usando imagens de satélite adquiridas em diferentes datas. Para isto, a compatibilidade espacial entre essas imagens é um requisito básico, mais ainda quando se trata de imagens de diferentes sensores. Nesse artigo é descrita uma metodologia para a semi-automação do processo de correção geométrica com a finalidade de facilitar o ajuste geométrico entre imagens de diferentes sensores. Inicialmente, uma imagem base é manualmente corrigida a partir de dados extraídos de uma carta topográfica digital. Após essa correção, a imagem corrigida é utilizada como elemento base de referência para corrigir as outras imagens (denominadas neste trabalho de imagens de ajuste), as quais são adquiridas em datas diferentes e/ou de diferentes sensores. Depois de corrigida a imagem base todas as imagens (base e de ajuste) são segmentadas e classificadas. Os segmentos classificados como vegetação florestal são escolhidos para compor a malha relacional. As imagens de ajuste são registradas pelo processo imagem-imagem usando os centróides dos
\end{abstract}


segmentos de vegetação florestal. Os segmentos de vegetação florestal presentes na imagem base são confrontados com os segmentos correspondentes nas imagens de ajuste, para buscar correspondências (matching). O processo de matching é realizado através da aplicação de algoritmos genéticos. Ao obter um resultado satisfatório na busca de correspondência, calcula-se os centróides correspondentes aos segmentos detectados, os quais são utilizados como pontos de controle para o processo de registro das imagens. Os resultados mostram que os algoritmos genéticos encontraram a solução ótima na maior parte dos experimentos realizados. Porém, para a imagem LANDSAT 2002 reamostrada a solução encontrada foi subótima, pois um segmento sofreu grandes variações em relação ao mesmo segmento na imagem base.

Palavras-chave: Registro de Imagem; Correspondência de Regiões; Algoritmos Genéticos.

\section{ABSTRACT}

This work is part of the GEOSAFRAS program - estimates and harvests in Brazil which is coordinated by the National Company of Supply and the United Nations Development Programme. A regular use in the management of harvests is the monitoring of the cultivation fields changes by using satellite images obtained at different dates. For this purpose, the spatial compatibility between these images is a necessary condition, even more when it comes to images from different sensors. This article describes a methodology for the semi-automation of the geometric rectification process aimed at facilitating the geometric adjustment between images from different sensors. First, a base image is manually rectified from data extracted from a digital topographic map. After such rectification, the correct image is used as a reference element to rectify other images (in this work, called adjustment images), which are acquired at other dates and/or from other sensors. Once the base image is rectified, all images (base and adjustment ones) are segmented and classified. The segments classified as forest vegetation are selected to compose the relational mesh. The adjustment images are registered by the image-image process by means of the forest vegetation segment centroids. The forest vegetation segments in the base image are compared to the corresponding segments in the adjustment images, to find pattern matching. The matching process involves the application of genetic algorithms. After obtaining a positive result regarding the pattern matching, the centroids corresponding to the detected segments are calculated; These centroids are used as control points for the image registry process. The results show that genetic algorithms have found the optimal solution in most experiments. However, regarding the LANDSAT 2002 resampled image, the solution found was suboptimal because one of the segments showed large variations in relation to the same segment of the base image.

Keywords: Image Registration; Region Matching; Genetic Algorithm. 


\section{INTRODUÇÃO}

No presente existe uma grande oferta de imagens adquiridas por plataformas orbitais, o que facilita o acompanhamento das mudanças que ocorrem temporalmente nos campos de cultivo. Porém, para que seja possível usar corretamente essas imagens é necessário que exista compatibilidade espacial entre as cenas capturadas em diferentes datas e por diferentes sensores. A compatibilidade espacial é obtida pela aplicação de métodos de ajuste geométrico (ou correção geométrica) entre diferentes imagens adquiridas de uma mesma região.

$\mathrm{O}$ ajuste geométrico de uma imagem é parte fundamental do processamento digital de imagens, ou seja, sem esse ajuste é muito difícil extrair informações métricas das imagens. O processo tradicional utilizado para fazer uma correção geométrica considera, num primeiro momento, a identificação de feições rígidas que possam ser usadas como elementos de controle, tais como: intersecções de estradas, delta de rios, entre outros. O problema dos processos de ajuste geométricos está na realização da identificação desses elementos rígidos apropriados de forma interativa (usuários localizam nas imagens e informam para o sistema a região apropriada). É necessário que esse processo seja automatizado ou, pelo menos, semi-automatizado e assim reduzir o tempo e os custos de processamento das imagens. Fonseca (2000) fez o registro automático de imagens baseando-se nos valores de níveis de cinza das imagens e nos máximos locais da transformada Wavelet. Já Fedorov (2002) realizou o registro semi-automático utilizando três métodos: o primeiro, misto com avaliação de transformação, o segundo baseado na transformada Walevet e o terceiro baseado em contornos. E Dal Poz e Scalco (2006), realizaram a orientação semi-automática de imagens CBERS usando rodovias como controle de campo, neste processo utilizou-se a DLT (Direct Linear Transformation) para fazer as correspondências pontuais. Entretanto, nas imagens de regiões com campos de cultivo pode não haver cruzamentos de estrada ou mesmo estradas bem definidas que possam servir de referência para o registro de imagens de diferentes épocas.

Este artigo apresenta uma proposta metodológica baseada na aplicação de algoritmos genéticos para analisar malhas relacionais, com o objetivo de encontrar regiões homólogas específicas, existentes em imagens adquiridas de uma mesma região, que contemple áreas de atividade agrícola e que contenha áreas de vegetação permanente ou áreas de preservação. Em regiões de atividade agrícola intensa as áreas de vegetação ocupam pequenas áreas remanescentes que mudam muito pouco com o passar dos anos, em contraste com os campos agrícolas, que sofrem intensas variações em seus padrões espaciais e espectrais.

A proposta deste trabalho é usar a propriedade de constância temporal na forma geométrica das áreas de vegetação, para identificar os centróides e os valores de área correspondente aos segmentos. Com esses dados extraídos é possível formar uma malha relacional, a qual servirá como elemento de apoio para a aplicação do método de ajuste geométrico. A malha relacional é construída tanto para uma imagem de referência como para uma imagem de ajuste. 
A partir das malhas relacionais extraídas faz-se a comparação entre os segmentos da imagem base com os segmentos das imagens de ajuste, para buscar a correspondência (matching) entres os segmentos (imagem base -> imagem de ajuste). A análise de correspondência é feita pela aplicação de algoritmos genéticos. Após haver sucesso na obtenção de elementos correspondentes, são extraídas as coordenadas dos centróides e das áreas em correspondência. Os valores desses centróides permitem ajustar geometricamente essas duas imagens e consequentemente ajustá-las.

\section{REVISÃO DE LITERATURA}

Segundo Duarte (2004) a interpretação estrutural de uma cena pode ser realizada a partir de um modelo esquemático genérico, ou seja, o reconhecimento de uma imagem pode ser visto como o processo de associação entre regiões (objetos) da imagem e objetos presentes no modelo esquemático utilizado. Este conceito implica na identificação da relação entre as regiões e os objetos no modelo, um problema que pode ser resolvido pela aplicação de técnicas de matching. Pode-se definir matching como sendo o estabelecimento de correspondências entre vários conjuntos de dados associados com imagens, mapas, modelos de objetos e dados de Sistemas de Informações Geográficas (SIG) (HEIPKE, 1997).

A identificação de objetos por uma malha relacional apresenta os seguintes princípios:

(a) Construção do modelo relacional para a classe de objetos;

(b) Construção da descrição relacional da imagem;

(c) Cálculo do isomorfismo (melhor correspondência parcial) entre a imagem e o modelo.

A Figura 1 ilustra um exemplo de duas malhas relacionais que contêm elementos que se correspondem, derivados de duas imagens adquiridas de diferentes visadas. Os modelos 1e 2 são, respectivamente, as malhas relacionais geradas a partir dos segmentos das imagens 1 e 2. Nesse caso o matching é feito pela comparação entre as malhas relacionais para se determinar a correspondência entre os elementos correspondentes nas imagens (nós (Q,T,E,E) dos grafos obtidos).

Figura 1 - Identificação por Malha Relacional.

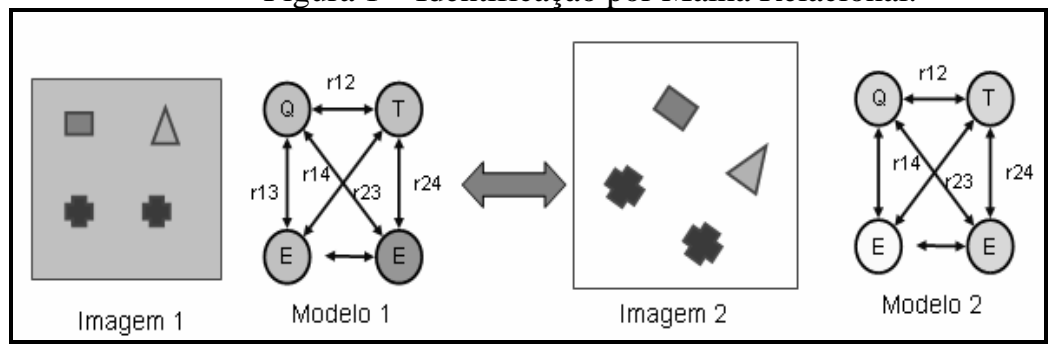

Bol. Ciênc. Geod., sec. Artigos, Curitiba, v. 17, nº 1, p.37-52, jan-mar, 2011. 
Aksoy (2006) relata que a relação espacial entre os elementos pode ser baseada na:

(d) Razão do limite comum entre duas regiões, ou seja, sobrepondo os elementos por meio dos seus perímetros;

(e) Distância entre duas regiões; e no

(f) Ângulo entre o eixo horizontal e a ligação de linha ao centróide dos elementos.

O problema central, neste caso, é a determinação dessa correspondência de forma automática. Uma solução possível é executar uma busca exaustiva entre todas as regiões possíveis e, mensurar o grau de similaridade entre elas e, optar por aquela que produzir o maior grau de similaridade. Na verdade, após montadas as estruturas das malhas relacionais o problema passa ter características de processos de otimização, para os quais podem ser aplicados algoritmos genéticos na determinação automática da existência, ou não, de correspondência de regiões.

\subsection{ALGORITMOS GENÉTICOS}

Algoritmos Genéticos são métodos de pesquisa estocástica na determinação de uma solução ótima ou subótima baseados no mecanismo de seleção e evolução natural (GOLDBERG, 1989). Segundo Luger (2004), os algoritmos genéticos são baseados numa metáfora biológica: a otimização como uma competição evolutiva de uma população de soluções candidatas à solução do problema.

A idéia central dos algoritmos genéricos é encontrar uma solução ótima combinando uma população de possíveis soluções dentro de um processo iterativo. Em cada passo deste processo as melhores soluções são identificadas e utilizadas na iteração seguinte, de forma similar a um processo evolutivo, onde os indivíduos mais aptos ao meio sobrevivem. A adaptação ou não ao meio em questão, é representada matematicamente por uma função de aptidão que atribui a cada indivíduo (possível solução) um valor em função de sua adequação para a solução do problema proposto. As possíveis soluções escolhidas são combinadas para gerar novas possíveis soluções e assim completar o número de possíveis soluções que foram descartadas (completar com uma nova população). Por meio de operações análogas à transferência de genes na reprodução natural, o método gera uma nova população de possíveis soluções combinando aquelas mais aptas obtidas na iteração anterior.

A utilização dos algoritmos genéticos se justifica para a solução de problemas para os quais é difícil, ou impossível, encontrar-se uma solução matemática única. Nesses casos, por ser difícil determinar a solução do problema, a opção é buscar uma solução por meio de processos de otimização. Para utilizar os algoritmos genéticos o problema deve apresentar as características descritas a seguir:

- O problema é modelado matematicamente e sua solução é representada como um conjunto de números a serem determinados; 
- A série de números associados a uma provável solução é representada como uma cadeia binária, imitando uma cadeia genética. Essa cadeia descreve um "indivíduo";

- Como a solução correta é desconhecida, um conjunto grande de possíveis soluções é inicialmente proposto, de forma aleatória, formando uma "população".

- Uma função de aptidão é proposta para descrever o grau de validade da solução, ou sua aptidão para a solução do problema. Fazendo a analogia genética, a função de aptidão descreve a aptidão do indivíduo para sobreviver no meio;

- Com essa população, o processo evolutivo é iniciado. Esse processo assume a forma de um processo iterativo, no qual as melhores soluções são preservadas e combinadas para gerar novas soluções, teoricamente mais adequadas. As piores soluções, pelo contrário, são descartadas. O processo, teoricamente, deve convergir para uma solução ótima ou subótima;

O processo iterativo termina quando um critério de aptidão é satisfeito pelo indivíduo mais apto, ou por um subconjunto de indivíduos mais aptos, ou quando um limite de número de iterações é atingido. Em seguida a solução mais apta é decodificada para se obter o conjunto de números da solução ótima para o problema.

O processo iterativo de evolução é regido por regras que definem a taxa de seleção, ou quantidade de sobreviventes de uma geração, o método de cruzamento e a ocorrência de eventuais mutações.

A seleção, dentro dos algoritmos genéticos, é artificial e obedece a regras fixadas pelo usuário. Sua função é escolher, dentro do conjunto de soluções disponíveis, um subconjunto de soluções para a geração de uma população, a ser utilizado em uma seguinte iteração. Entre as técnicas de seleção mais utilizadas podem ser citadas: elitismo, por sorteio (roleta) e torneio (GOLDBERG, 1989).

Nesse trabalho foi utilizada a seleção por elitismo, na qual os indivíduos devem ser ordenados de forma decrescente de acordo a sua aptidão e os indivíduos com valores mais altos são selecionados. O elitismo é a técnica mais utilizada para melhorar a convergência dos algoritmos genéticos. É uma adição aos métodos de seleção que força os algoritmos genéticos a preservar certo número de "melhores" indivíduos em cada geração. Tais indivíduos podem ser perdidos se não forem selecionados para reprodução ou se forem destruídos por cruzamento ou mutação. Em outras palavras, o elitismo seleciona os melhores cromossomos de uma população e transporta-os à geração seguinte. Quando a técnica do elitismo é utilizada, o algoritmo converge mais rapidamente. Em geral a elite tem um tamanho reduzido, em torno de $4-5 \%$ pois se for muito grande, corre-se o risco de que a população convirja para uma população homogênea, não explorando outras soluções. A seleção por elitismo depende da função de aptidão que guia o processo 
na direção do objetivo desejado. Quanto maior o valor de aptidão de um indivíduo, maior será a adequação desta série de números para a solução do problema.

Os indivíduos selecionados são submetidos ao processo de cruzamento. Este processo consiste em escolher dois indivíduos e, a partir de sua cadeia genética, gerar novos indivíduos para uma nova geração. Para a escolha dos indivíduos que serão usados no cruzamento, pode-se utilizar as técnicas sorteio ou torneio.

- $\quad$ Na técnica de seleção por sorteio (roleta), dois indivíduos são escolhidos aleatoriamente.

- A seleção por torneio consiste em comparar a aptidão de um subconjunto, escolhido aleatoriamente, e selecionar o indivíduo mais apto dentro deste conjunto. Este processo é repetido várias vezes até atingir o número desejado de indivíduos selecionados.

Uma vez executada a seleção, o conjunto de indivíduos selecionados dá origem à nova geração. Como o conjunto selecionado é menor que o tamanho da população utilizada, novos indivíduos são produzidos através do cruzamento dos indivíduos selecionados. O processo de geração de novos indivíduos obedece a leis propostas pelo usuário e são descritas como operadores genéticos de cruzamento (cross-over) e mutação.

Os Operadores Genéticos, mutação e cruzamento, têm por função, segundo Goldberg (1989), reger a evolução da espécie. No cruzamento, duas cadeias genéticas selecionadas trocam material genético entre si gerando novas cadeias híbridas, conforme mostra a Figura 2.

Figura 2 - Cruzamento.

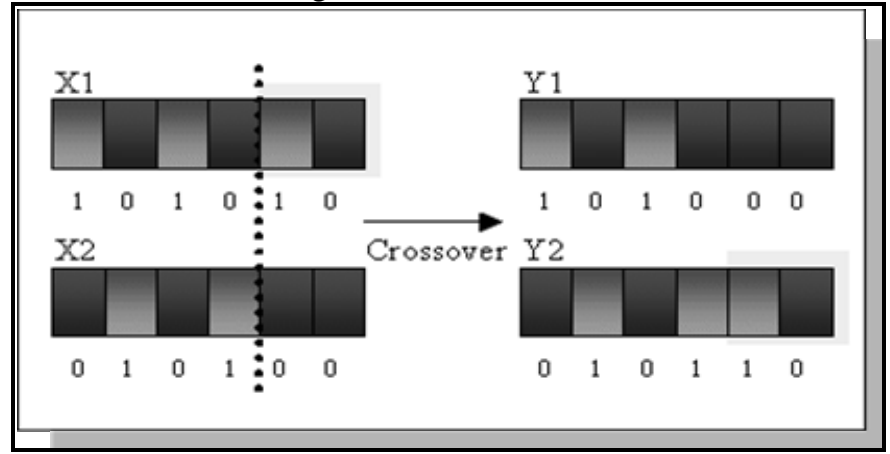

FONTE: LIAO e SUN (2001)

A mutação atua alterando o código genético, ou seja, um ou mais genes são modificados no cromossomo pai gerando um filho mutante, conforme pode ser observado na Figura 3. 
Figura 3 - Mutação.

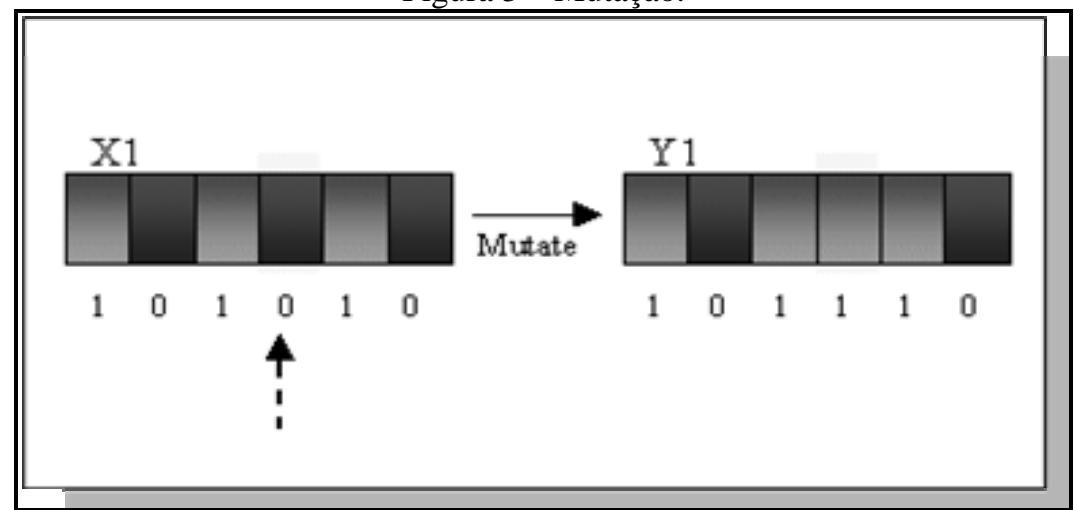

FONTE: LIAO e SUN (2001)

\section{METODOLOGIA}

Na análise de correspondência uma das imagens é adotada como referência, sendo manualmente corrigida a partir de dados extraídos de uma carta topográfica digital. No processo de registro imagem-imagem são usadas duas imagens de uma mesma região.

Inicialmente, as imagens são segmentadas e classificadas. A segmentação e classificação foi efetuada no software eCognition. Não são abordados detalhes dessas etapas neste artigo. Do resultado da classificação, foram separados apenas os segmentos classificados como vegetação florestal para a análise de correspondência.

Na imagem de referência os segmentos classificados como área de vegetação florestal são codificados e é construída uma malha relacional (grafo do relacionamento espacial) dos segmentos escolhidos (Figura 4a). Esse mesmo processo é aplicado numa segunda imagem, a qual deve ser geometricamente ajustada em relação à geometria da primeira imagem (Figura 4b). Os segmentos de vegetação utilizados para fazer essa correspondência são descritos pelas coordenadas de seus centróides, suas áreas e um valor de rótulo e a distância entre segmentos.

O procedimento de otimização é definido pelo processo de busca de uma malha relacional semelhante ou igual à malha de referência, definida previamente na imagem de referência. Para solucionar o problema com aplicação de algoritmos genéticos, é necessário: 1) fazer a codificação dos indivíduos; 2) definir a população; 3) selecionar operadores genéticos; e 4) descrever a função de aptidão (fitness). Estas etapas estão descritas a seguir. 
Figura 4 - Exemplo de Malhas Relacionais de Duas Imagens.

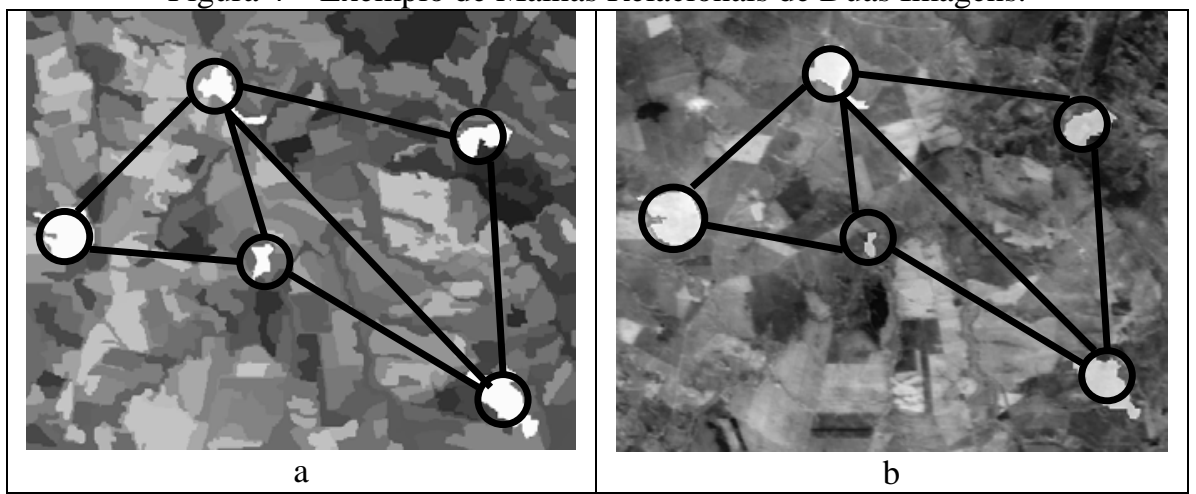

\subsection{Codificação do Problema e dos Indivíduos}

Basicamente, o problema consiste em associar um conjunto de " $n$ ” segmentos na imagem de ajuste a "m” segmentos na imagem de referência. A solução pode ser representada como vetor $n$-dimensional, onde o valor armazenado em cada posição do vetor descreve um segmento associado a um segmento na imagem de ajuste. A Figura 5 ilustra o caso em que o vetor de solução (codificação) tem seis elementos e, cada um deles armazena um valor de rótulo que associa o segmento representado no vetor com o segmento correspondente na imagem original. O vetor solução é vs' $=[3,1,1,2,4,5]$. Quando não é possível estabelecer uma relação para um segmento, então ele é rotulado com o valor zero.

Figura 5 - Possível Associação entre a Imagem de Referência e Imagem Ajuste.

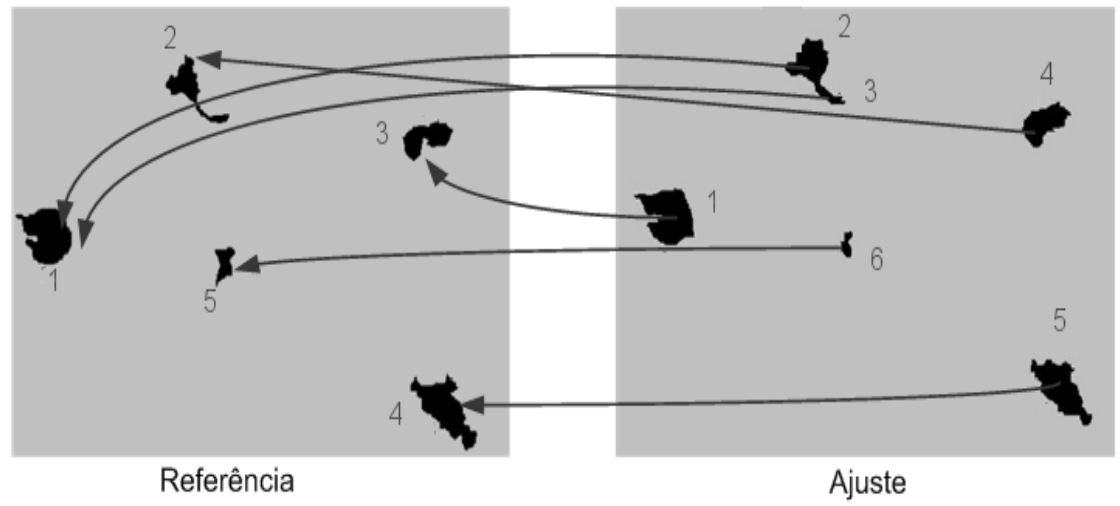

Bol. Ciênc. Geod., sec. Artigos, Curitiba, v. 17, nº 1, p.37-52, jan-mar, 2011. 
O conjunto de valores da solução são números inteiros positivos. Tais números são codificados como uma série de valores binários. A codificação binária é muito usada em algoritmos genéticos (Mitchell, 1997) devido à sua simplicidade computacional. O tamanho da cadeia depende da representação dos números inteiros, neste caso, de oito bits.

A população inicial é criada de forma aleatória. Para cada gene no indivíduo escolhe-se, aleatoriamente, o número 1 (um) ou 0 (zero) até preencher todos os indivíduos da população.

\subsection{Função de Aptidão (FITNESS)}

O objetivo da análise de correspondência entre segmentos é a identificação dos segmentos homólogos, logo, considerou-se que a função de aptidão deveria refletir dois aspectos: a similaridade entre os segmentos homólogos e a similaridade entre a distribuição espacial dos segmentos. Assim sendo, a função de aptidão (FA) foi definida como sendo a soma ponderada de dois fatores, conforme equação 1 .

$$
F A(x)=a * F S+(1-a) * F E
$$

Onde:

FS mede a similaridade entre os segmentos; e

FE descreve a similaridade da distribuição espacial dos segmentos.

Para o cálculo de FS, utilizou-se a comparação do tamanho dos segmentos nas duas imagens. Considerando o tamanho do segmento (S), um valor para o fator FS é obtido a partir da diferença de tamanho médio (DMS) entre os segmentos da imagem base referência e os segmentos da imagem de ajuste, em acordo com a equação 2:

$$
D M S=\left|\frac{S_{R i}-S_{A i}}{\sum_{k=1}^{n} S_{R i}}\right|
$$

Onde:

$S_{R i}$ é o tamanho do segmento "i” na imagem de referência (em pixel);

$S_{A i}$ o tamanho do segmento "i" na imagem ajuste (em pixel) e

$n$ é o número total de segmentos.

O valor ideal na diferença de tamanhos dos segmentos é zero. Valores pequenos podem ser considerados também adequados. Analisando a equação 3 percebe-se que à medida que a diferença cresce, o valor deste fator deve diminuir, reduzindo a aptidão do indivíduo. Assim sendo, uma função decrescente é usada para normalizar o valor de aptidão, como mostra a Figura 6 e a equação 3. 
Figura 6 - Obtenção de FS por Normalização De DMS.

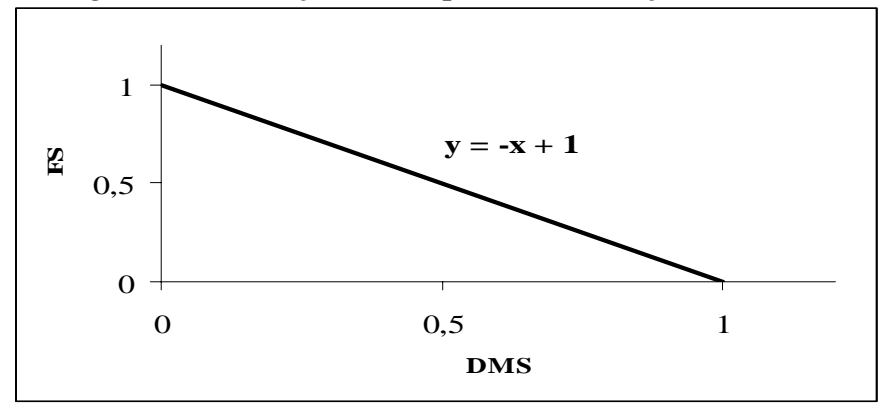

$$
F S=1-D M S
$$

De maneira similar, o fator $F E$ é calculado comparando as distâncias que separam os centróides dos diferentes segmentos em cada imagem. Para isso, uma matriz de distâncias entre os centróides dos segmentos é calculada para a imagem de referência, levando em consideração eventuais diferenças de resolução espacial. No processo iterativo, para cada solução, uma matriz similar é calculada e comparada com a matriz de referência. A comparação é feita pela diferença destas duas matrizes.

A distância entre dois segmentos na imagem de referência é obtida utilizando a equação 4 (distância euclidiana):

$$
\operatorname{Dr}^{2}(i, j)=\left(x_{R i}-x_{R j}\right)^{2}+\left(y_{R i}-y_{R j}\right)^{2}
$$

Onde:

$\left(x_{R i}, y_{R i}\right)$ representam as coordenadas do centróide do segmento " $i$ " na imagem de referência;

$\left(x_{R j}, y_{R j}\right)$ representam as coordenadas do centróide do segmento “j” na imagem de ajuste.

De forma análoga, a distância na imagem ajuste é obtida pela equação 5:

$$
D a^{2}(i, j)=\left(x_{A i}-x_{A j}\right)^{2}+\left(y_{A i}-y_{A j}\right)^{2}
$$

Onde:

$\left(x_{A i}, y_{A i}\right)$ representam as coordenadas do centróide do segmento "i” na imagem de ajuste;

$\left(x_{A j}, y_{A j}\right)$ representam as coordenadas do centróide do segmento “j” na imagem de ajuste. 
O valor médio da diferença normalizada entre as distâncias é calculado pela equação 6:

$$
D M E=\left|\frac{\operatorname{Dr}(i, j)-D a(i, j)}{\sum \operatorname{Dr}(i, j)}\right|
$$

Onde:

$D M E$ é o valor médio da diferença de distância normalizada entre os segmentos "i” e "j".

O valor ideal do $D M E$ é zero. Considerando também que na medida em que a diferença de distância normalizada $|\operatorname{Dr}(i, j)-D a(i, j)|$ aumenta, o valor do fator $F E$ (equação 7) deve diminuir, o que determina uma função decrescente similar à apresentada na Figura 6, o termo $\sum \operatorname{Dr}(i, j)$ é usado para normalizar o valor do $D M E$. O valor $F E$ é, então, calculado a partir da equação 7.

$$
F E=1-D M E
$$

\section{EXPERIMENTOS}

Nos experimentos realizados para avaliar a metodologia proposta foram utilizadas imagens multitemporais da região do município da Lapa, no Estado do Paraná, cuja localização está apresentada na Figura 7. O conjunto de dados inclui: imagens adquiridas pelo satélite CBERS II/CCD com resolução espacial de 20 metros ( novembro de 2004, e janeiro e agosto de 2006); imagens ASTER com resolução espacial de 15 metros ( maio de 2003 e abril de 2006); uma imagem LANDSAT com resolução espacial de 28,5 metros (julho de 2000) e uma imagem LANDSAT com resolução espacial de 30 metros (dezembro de 2002).

Figura 7 - Área de Estudo.

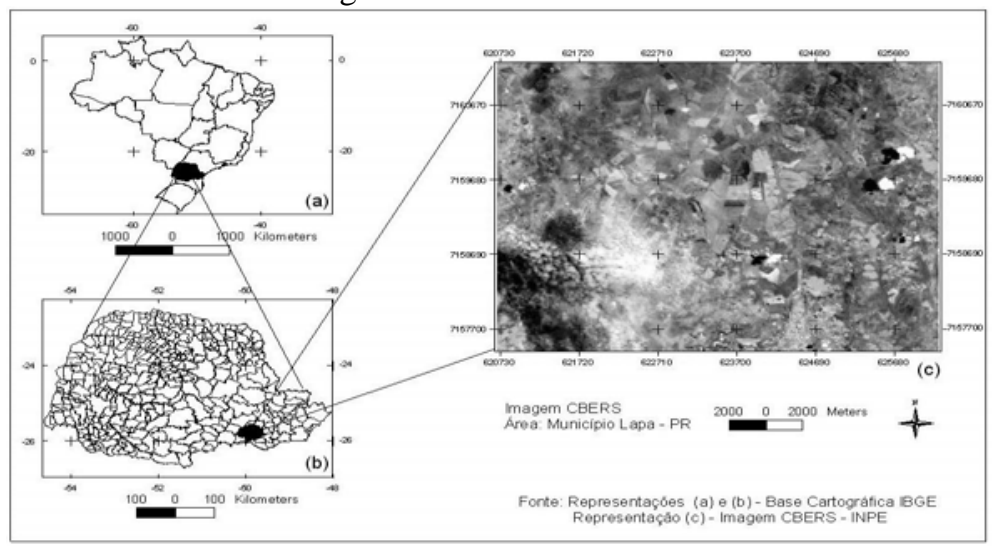

Bol. Ciênc. Geod., sec. Artigos, Curitiba, v. 17, nº 1, p.37-52, jan-mar, 2011. 
Como imagem de referência foi adotada a imagem CBERS de janeiro de 2006. As imagens dos outros sensores foram reamostradas para compatibilizar a dimensão do pixel com a resolução espacial da imgem CBERS. Devido às diferenças espectrais e espaciais entre as imagens, o número de segmentos na imagem de ajuste é muito maior que o número de segmentos na imagem de referencia.

Uma série de experimentos foi efetuada variando a quantidade de regiões utilizadas. No primeiro experimento foram utilizados todos os segmentos disponíveis na imagem de ajuste. Com o uso de todos os segmentos não se conseguiu obter resultados satisfatórios e, além disso, há a implicação de um maior custo computacional. Nos experimentos a seguir a quantidade de regiões foi reduzida para cinco ou oito segmentos. Essa redução foi feita por meio do agrupamento segmentos contíguos, para formar elementos maiores, obtidos no resultado da segmentação. Desses agrupamentos foram computados os respectivos valores de área e coordenadas dos centróides.

\section{RESULTADOS E DISCUSSÕES}

Utilizando todos os segmentos, a solução ótima ou subótima da correspondência ideal para cada imagem de ajuste é diferente, pois o resultado do processo de segmentação não é o mesmo para todas as imagens de ajuste. Para a imagem LANDSAT 2002 foi encontrada a solução subótima, e para as demais imagens foi encontrada a solução ótima da correspondência ideal da malha.

Quando são utilizados todos os segmentos da imagem de ajuste, a quantidade de elementos presentes nesta é maior do que os presentes na da imagem de referencia. Para que o vetor da solução tivesse o mesmo número de elementos, houve necessidade de combinar regiões da imagem de ajuste. Por exemplo, na imagem ajuste CBERS II 2004, os segmentos 1, 2, 3 e 4 foram subdivididos em três partes respectivamente, conforme mostra a Figura 8. Então, a solução ótima neste caso é [1 1111222223333444 5].

Figura 8 - Correspondência da Malha para os Segmentos Subdivididos.

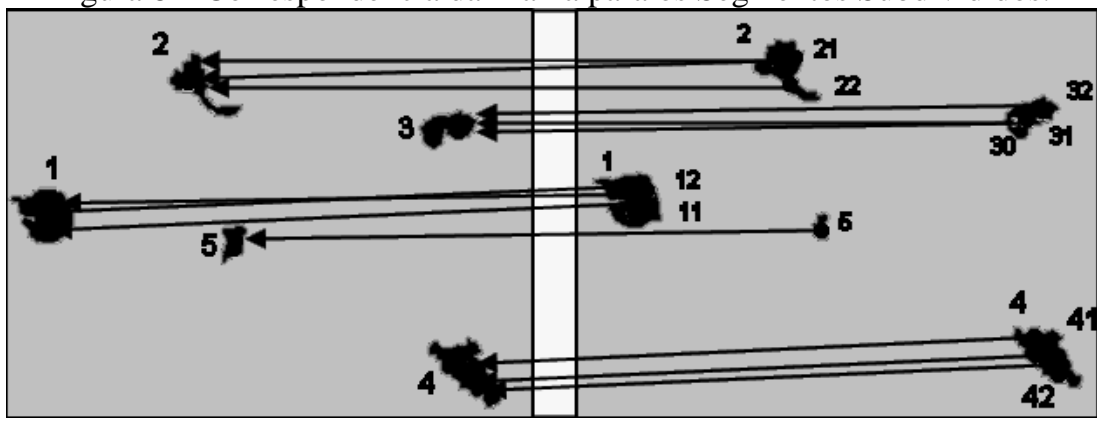


No segundo experimento, cinco regiões foram consideradas. A solução ideal é mostrada na Figura 9. Somente em um caso, para a imagem LANDSAT 2002, os algoritmos genéticos encontraram uma solução subótima e não uma solução ótima. No caso da imagem Landsat de 2002, não foi possível encontrar o segmento de identificador 3 na imagem de ajuste, pois ele representa apenas $31 \%$ da área do mesmo na imagem de referência.

Figura 9 - Correspondência da Malha para Cinco Segmentos.

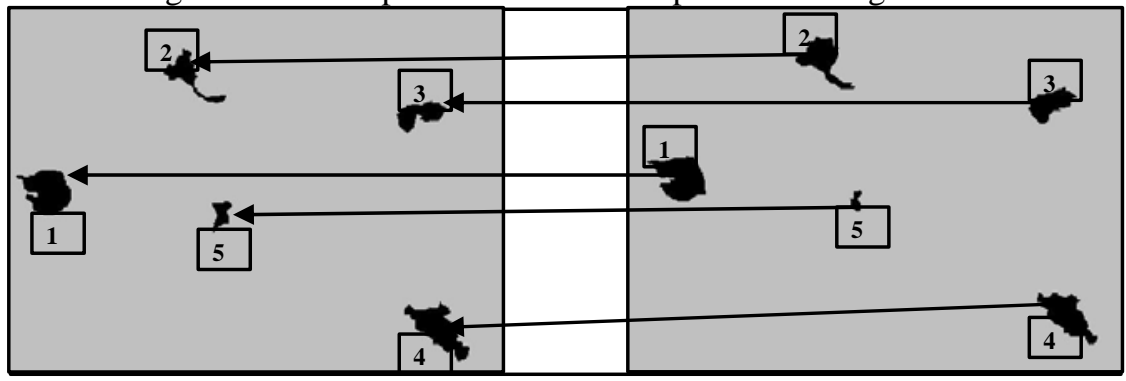

No terceiro experimento foram utilizados oito segmentos, conforme pode ser observado na Figura 10, sendo que cinco deles os mesmos utilizados no segundo experimento. Para esse experimento foi possível encontrar a solução ótima para todas as imagens de ajuste.

Figura 10 - Correspondência da Malha Relacional para Oito Segmentos.

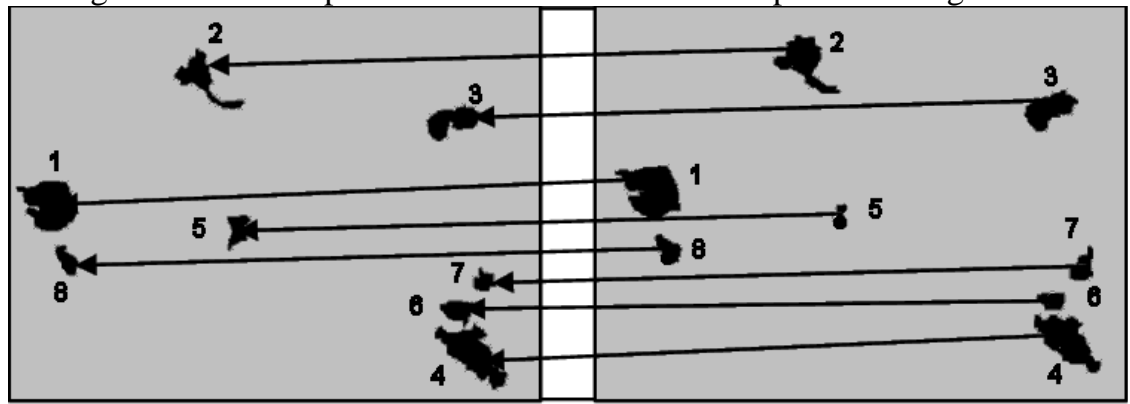

\subsection{Registro das Imagens}

Finalmente, usando os centróides das regiões homólogas identificadas, as imagens de ajuste foram compatibilizadas geometricamente com a imagem de referência. A compatibilização geométrica das imagens foi realizada a partir dos centróides dos cinco e oito segmentos. Os melhores resultados foram obtidos usando cinco centróides na transformação afim. Quando se utilizou oito centróides 
foi possível verificar a existência de pontos que introduzem erros grandes no modelo. Assim sendo, considerando a existência de uma quantidade relativamente grande de centróides, foi possível retirar até três pontos, procurando assim melhorar o modelo e reduzir o RMS (Erro Médio Quadrático) para menor que um pixel.

Dentro destas condições, para as imagens CBERS II 2004, LANDSAT 2000 e LANDSAT 2000 reamostrada, LANDSAT 2002, ASTER 2003 reamostrada, ASTER 2006 e ASTER 2006 reamostrada foi possível obter o RMS menor que um pixel, ou seja, um registro dentro do padrão esperado.

\section{CONCLUSÕES}

A metodologia proposta, para efetuar o registro de imagem-imagem por correspondência (matching), baseada nos algoritmos genéticos, foi eficiente. Principalmente na busca da solução ótima ou subótima de correspondência da malha relacional, tanto para as imagens de ajuste, com mesma resolução espacial da imagem de referência, como para as imagens com resolução diferente.

A viabilidade do uso de centróides como pontos de controle foi comprovada, mas que é preciso que os segmentos utilizados tenham uma boa distribuição espacial na imagem. Ou seja, é necessário que a distribuição espacial dos centróides dos segmentos de vegetação forneça uma boa geometria para realizar a correção geométrica. Quando os segmentos não possuem uma boa distribuição espacial pode-se atribuir mais pontos de controle, o que permite melhorar a distribuição dos pontos na imagem, levando-se a obter um melhor resultado da compatibilização geométrica de imagens adquiridas em datas diferentes ou de diferentes sensores.

\section{REFERÊNCIAS BIBLIOGRÁFICAS}

AKSOY, S. Modeling of remote sensig image content using attributed relational graphs. In Proceedings of IAPR International Workshop on Structural and Syntactic Pattern Recognition v 4109, p 475-483, 2006.

DAL POZ, A. P.; SCALCO, J. C. Orientação semi-automática de imagens CBERS usando rodovias como controle de campo. Boletim de Ciências Geodésicas V. 12 n 2, p 295-313, 2006.

DUARTE, A. R. Novas heurísticas e uma abordagem por programação inteira para um problema de correspondência inexata de grafos. 85f. Dissertação (Mestrado em informática) - Departamento de informática, Pontifícia Universidade Católica do Rio de Janeiro. Rio de Janeiro. 2004.

FEDOROV, D. Sistema semi-automático de registro e mosaico de imagens. $147 \mathrm{f}$. Dissertação (Mestrado em Computação Aplicada) - Instituto Nacional de Pesquisas Espaciais. São José dos Campos. 2002.

FONSECA, L. M. G. Registro automático de imagens de sensoriamento remoto baseado em múltiplas resoluções. 219f. Tese (Doutorado em Computação Aplicada) - Instituto Nacional de Pesquisas Espaciais. São José dos Campos. 2000. 
GOLDBERG, D. E. Genetic algorithms in search, optimization and machine learning. Editora: Addison-Wesley. New York 1989. 412 p.

HEIPKE, C. Automation of interior, relative, and absolute orientation. ISPRS Journal of Photogrammetry \& Remote Sensing v. 52, n. 1, p.1-19,1997.

LIAO, Y.-H.; SUN, C.-T. An Educational Genetic Algorithms Learning Tool. IEEE Transactions on Education, v. 44, n. 2, 2001. htttp://ewh.ieee.org/soc/es/May2001/14/Begin.htm

LUGER, G. F. Inteligência artificial: estruturas e estratégias para a solução de problemas complexos. Tradução Paulo Engel. 4 ed. Porto Alegre. Bookman, 2004. $774 \mathrm{p}$.

MITCHELL, M. An introduction to genetic algorithms. Cambridge: Mit Press, 1997. 207 p.

(Recebido em abril de 2010. Aceito em fevereiro de 2011) 